\title{
LOBBYING FOR VALUE -
}

\section{A DIALOGUE}

\section{Keywords}

Labor

Profession

Market

Discipline

Added value
Through the claim that architects are precarious workers, the Architecture Lobby gathered and organized to demand a fair valuation of architects' services. In this conversation between its members, however, they go beyond the architect and deepen into notions of architectural value which end up unpacking the whole system that surrounds our work.

PD: In the call for articles on 'Value,' ARQ editors state that architectural value is caught between being understood as a concrete 'use value' - a synthesis of economic variables (land, material, square footage) and an 'exchange value' that is determined not by need/use but by transactional agreements of a social nature. They hint at the fact that 'architectural value' might be (but is not) assigned to 'use value' though they are not clear if they want to suggest that - whatever 'architectural value' is - would be an aspect of 'exchange value.'

There is much to be said about both these questions: do we really believe, even in the most real estate/development definition of architecture, that use value is determinable? Or more basically, does the concept of use value have any meaning since the Industrial Revolution?

However, I want to begin with how the call is framed in general; that is, linking the economic question to an ethical one. What, they ask, are the links between a building's value and our own disciplinary sense of what matters when we engage in our métier? What values do we hold? I am interested in this elision as a starting point because in the description of the Architecture Lobby's work, we say: "The Architecture Lobby is an organization 
Participantes (en orden de aparición, todos

miembros de Architecture Lobby)

Participants: (in order of appearance; all are

members of the Architecture Lobby)

Peggy Deamer PD

James Heard JH

Aaron Cayer AC

Phil Bernstein PB

William Huchting WH

Gabriel Cira GC

Manuel Shvartzberg MS

Marianela D'Aprile MDA

David Hecht DH

of architectural workers advocating for the value of architecture in the general public and for architectural work within the discipline." We offer, I suggest, the same elision of ethical value with monetary value, an elision that I feel allows us to be fuzzy about precisely what we are proposing. Are we talking about money - for owner, designer and/ or worker - or are we talking about the social value towards which our ethics are pointed?

Maybe this isn't such a problem: the term 'added value' - which architecture seemingly brings to building - might happily synthesize this elision in suggesting that the social and aesthetic knowledge we 'add' will (hopefully) be understood by the public and (hopefully) be returned to us via compensation. My hope is that Lobby members' thoughts on this will circle back to the original questions of use and exchange value in architecture.

JH: Peggy, rather than approaching architecture from the use/exchange distinction of commodities, I would address it as an issue of valorization vs fetishization; that is, if architecture finds itself in a state of arrested fetishization, it may be that the immediacy of the commodity blinds us from the more pertinent place to look.

This means focusing the discourse on the ability of architecture to conjure value through 'place' as opposed to the 'thing.' Exchange value, as the name would suggest, is shown through the exchange of the thing, and hence addresses the wrong thing.

I would argue that the exchange of architecture is fundamentally distinct from that of other goods in that it is rooted to place and cannot be physically exchanged. Rather than a good, it is a territory that changes hands, and the valuation of territory in late capitalism is directly tied to the speculative value of that land. In a speculative scheme, architecture is judged in relation to its ability to extract the 
potential latent in the territory, a potential that is dictated by a market; it is relied upon as a means of rent extraction. All this is to say that architecture itself is not the commodity, but a valorizing act upon land itself.

Just to be clear on the terms and thoughts here: I'm using 'commodity' and 'commodification' in the Marxist sense, as an object that has a use (material) value recognized at creation and an exchange (social) value recognized during exchange in the market. As there is certainly social value attached to architecture, the needle I'm trying to thread is whether that social value is the one that obfuscates labor (and therefore an exchange value), or rather a separately assessed judgment.

In any case, architectural value is not equivalent to exchange value; rather, it has to do with its capacity to valorize. Architecture recognizes value in place, and if it ceases to extract or maximize that value, it will be demolished without hesitation.

AC: This is interesting, but it circles back to a historical position of architectural value, by focusing on the object as the site of value rather than the labor of the architect - a position which the Lobby has tried to distance itself from. By re-thinking and re-positioning value very specifically in terms of 'work' (from knowledge to models to documents) rather than the so-called final object, architects are tasked to position themselves in the existing political economy, rather than pretend that they and their objects are immune to it. So the question is, what is the value of architectural 'work'? If architects were to imagine not being paid through a percentage of construction or by hourly wage, how might they make a claim for alternative forms of compensation that suggest value in terms other than money?

PB: In as much as I'm following, I would ask whether we can reduce the 'value' of all buildings to their 'valorized result' using exclusively the construct of the marketplace. That model works quite well for a commercial building, which only comprises a part of all buildings designed by architects. But what about a public library, a school, a non-profit 
health clinic where the transactional objective isn't determined by the market? I would argue that transaction is quite different for, say, a skyscraper in New York and an out-patient outreach clinic run by an NGO somewhere.

WH:The evaluation of architectural labor - and value are market driven, but what market driven means is very indeterminate and connects to how autonomous or not architecture and architectural labor are conceived. For example: I have a friend who is a professional artist. In a good year, he makes well into the six figures selling his work at Art Miami and Art Basel. I designed two homes and studios for him. Neither went through. His unvarnished reaction was "Why should I pay you then?" I made art for him and outside the built realm it has no value, he says. But consider: he makes USD 2,500 worth of materials into an artwork that his gallery sells in a series of 8 at USD 35,000 apiece; they sell out. Does the work have value outside the connections that his gallery has? Neither the art world nor architecture is interested in labor. They care about the end product valued as an investment. This is the nature of the market place.

PD: Just some thoughts to bring the discussion back to Aaron's point; I too am surprised that the conversation is focusing so much on the object/ building, even if we see 'it' in terms of social, use, valorization, or exchange value. To back up: the example that William brings up gives an accurate picture of how the public and our clients see us, and hence how our work is (very sadly) valued. But the point for me (and Aaron) is that this line of reasoning assumes that we (architects/Lobby members) agree that the thing being valued is the architecturally designed object even if we disagree with how it is assigned value. I (and Aaron) believe instead that the object is the tip of the iceberg, the physical evidence of an astounding array of knowledge that is really what we offer (I hesitate to use the word 'produce'). As long as we believe that what we do is design buildings - as opposed to offering knowledge about a large array of things, not the least of which is

"As long as we believe that what we do is design buildings - as opposed to offering knowledge about a large array of things - we shouldn't be surprised that the public thinks that architects make objects and reads the most mundane criteria of 
weighing in on the program or site or need to build at all - we shouldn't be surprised that the public thinks that architects make objects and reads the most mundane criteria of deeming its value. If we can agree that we are knowledge producers, there is still, however, the question of how to give value to what we are offering/selling (knowledge). We should come back to that. But in the meanwhile, I like to think of the pill analogy: we don't pay for that little white object; we pay for all the medical research that went behind it.

The question is that of the 'social' value that this knowledge offers. One way that (I believe) Phil was suggesting, is that it comes in the form of program and our ability to materialize aspirations that are not merely mercenary. This is not insignificant and it comes from that body of knowledge that I am arguing for and that should be paid for. But I worry that this, too, is too object/building focused.

$\mathrm{JH}$ : Peggy, I'm in agreement with your outline of architectural value and your last points. As I read them, the issue at hand is one with capitalism itself. I don't believe it's a question of non-existence of a fundamental value that must be uncovered as much as it is an effort to fight against that which attempts to erase the fundamental value of architecture. As I see it, the flaw is on the effects of capitalism on architecture rather than architecture itself, and because of that I think it's important to escape the thing, to pull it apart, and address it as a series of relationships to understand how fundamental, or perceptual, values are obscured.

PD: James, I'm sure you are ultimately right; it's Tafuri's argument: every piece of architecture will contribute to the unjust work of capitalism until capitalism is overthrown. But besides this question of what 'escape' means, it is too depressing to think that we can't do better, even if 'better' is determined by our own sense of self-worth; even if we are not talking about monetary value but aligning our social (or whatever) aspirations with reality. But I think we could still do better even in the monetary game if we could prove the ultimate benefits of our work for the public/clients.

GC: I want to focus on the public space of vision. I use 'vision' broadly, to mean the set of ways that people experience buildings within context, in person but also through media and common narratives; the experience of a quasi-object/building/thing. Architecture concerns itself with the study and shaping of quasi-objects, not buildings. Actually, we are always talking about quasi-objects, not buildings or anything else - we draw from a reserve of communal knowledge that is created by experience (including documentation and all other types of media). 
"I like to think of the pill analogy: we don't pay for that little white object; we pay for all the medical research that went behind it."

Within this framework, here is a definition of architectural value: Architecture constitutes an exception to the fixtures of everyday life - this it shares with all other forms of art, spectacle, celebration, etc. The exception is the source of its power (to furnish an aesthetic experience) and the bracketing of that power.

Architecture immediately pronounces itself as architecture. Moments of fixed attention and recognition, reaffirming the object's identity as architecture, are a commodity among others in service of capital. This difference is asserted in many ways, often those which sacrifice other types of value in the name of this surplus. 'Difference' stands for an opposition between architecture and the fabric of the city, and the latter stands for its own indifference and the indifference of perception afforded in exchange. The disciplinary and professional value of architecture resides within this 'difference' and, unfortunately, it has become the expectation and the de facto value of architecture and architects. Hopefully it's possible to see otherwise.

Can we conceive an architectural value that functions without an enunciation of difference for the sake of difference? Can we kick our addiction to pure difference? One way to think about this is through the two senses of the word 'performance' (performance as in putting on airs and performance as a tool serving to its capacity). Unlike the opposition of 'usevalue' and 'exchange-value,' common narratives easily confuse types of performance within architecture... but both can have equal real-world effects by altering perception and then behavior. Architects shouldn't mine abstract value straight from difference, but rather focus on specific (social or 'public') value and use design to increase the likelihood that they will accrete around and within a quasi-object.

And here is where I start to see parallels to the vectors of The Architecture Lobby. 1) It is necessary to identify the value of architecture as civic maintenance - the maintenance of everyday life for all possible subjects. 2) It is necessary to recognize that the labor of architects may not be 'visible' as such, and precisely for this reason it must be supported rather than pinched. 3 ) It is necessary to see that architects should be 
evaluated by their service to the public space of vision rather than the private space of the market.

PD: Gabriel, I love the idea of the quasi-object that is architecture and that might be the place to lodge its value. What comes to mind for me is all the images, theories, histories, heroes, as well as the narratives that constitute what works in our imaginary. But if this is what you mean by a quasi-object, it speaks of a 'value' that we in the discipline acknowledge but not something that equates with monetary value as determined by the public/market. In which case, we are back to the same ethics vs. money ambiguity when defining value. But I am also intrigued by the character of the 'difference' that we now too easily go for and should be set aside for its subtler cohort. Can we say it is design virtuosity? Or am I giving it a too blunt characterization? In any case, I want to endorse the three arenas in which you locate value.

MS: As already mentioned, a fundamental question around value in architecture is that of constituency. In other words, asking 'who is architecture for?' is a reciprocal question to that of 'what is architecture?' - perhaps in a dialectically-entwined way. As William mentioned, this is another way of posing the perennial problem of architecture as an 'autonomous' discipline - or in Gabriel's parallel formulation, of how not to reify architecture's elitism (its selfproclaimed autonomy) as a producer of abstract 'difference' which is the very lifeblood of capital.

In historical terms, the emergence of architecture as a distinct discipline has everything to do with the professionalization of expert knowledge as a sector of the labor market. Concretely, this meant taking traditional practices of building and design, usually shepherded by guilds or other forms of association, and giving their exclusive use to a new group - i.e. architects or engineers - so that the latter group could develop the practices 'autonomously,' thus being able to charge a specialist's fee. This shift changed the monopoly of building knowledge and culture from relatively social and common structures (guilds) to liberal professionals (individuals and their private firms). The formalization of previously common forms of knowledge in the hands of a few served two purposes: architectural services could become both more modern (i.e. reliable, standardized, maximized, predictable, etc.), as well as distinguished from those of others (whether craftsmen, builders, engineers, artists, etc.), therefore maximizing the efficiency of the division of labor in society as a whole. As a result, if you were a buyer of labor in the market, rather than a seller, you had more, and cheaper, to choose from. 
The liberal division of labor emerged chiefly as an instrument of the bourgeoisie - those who could contract architects, engineers, and other specialists; a tool for the domination of social classes without capital, rather than for their emancipation.

Thus, when we discuss the 'value' of architecture as a socially-embedded practice we have to be aware that at the level of the division of labor of the built environment, our profession is primarily a bourgeois one. The 'value' our activity brings, inscribed in these systems of labor that are mediated by states, their laws, and the political infrastructures between them (today, heavily weighted by large corporations and vested interests), is intrinsically tied to a logic of market competition. The ultimate evolution of this logic when left to capitalism is one of survival of the fittest according to price. Thus, strictly speaking, our motto is a contradiction in terms: the goal of liberal professions in a market society is to attempt to monopolize services and/or become more efficient than others in a narrowly cannibalistic, competitive struggle, thus ultimately decimating the 'general public' we claim to be upholding.

In order to get out of this vicious cycle, we would have to try to reinvent the boundary between 'architecture' and those other professions/ systems that co-produce the built environment - in a cooperative rather than a competitive direction. This doesn't mean simply building more 'social' architecture - which is a great commodity for contemporary capital, as the architectural darlings of neoliberalism clearly show (like Aravena's practice Elemental, to give a Chilean example). We have to change the underlying logics of how the built environment is produced.

"When we discuss the 'value' of architecture as a socially-embedded practice we have to be aware that at the level of the division of labor of the built environment, our profession is primarily a bourgeois one."

Not only would architectural labor have to cease to be merely a commodity in the labor market, but its patrons would have to become primarily public entities rather than private ones.

In other words, the very activity of architecture as we know it would have to be radically changed. No longer would it be private firms competing for projects according to a market logic, but there would have to be a democratic infrastructure in 
place that would determine beforehand who does the projects and how. Or, to use Peggy's terms, substantive 'use-values' would have to replace market 'exchange-values' as the driving force in the delivery of architecture.

Given capitalism's proven ability to allocate resources brutally and effectively according to certain performance criteria (usually, low prices based on labor exploitation), it would take enormous socio-political pressure to achieve the leverage needed for this change.

The society we are talking about here would be less 'efficient' and 'innovative' - in the extreme ideological terms that are shoved down our throats every day. But it would allow for the expression of other forms of efficiency, innovation, and creativity to surface - more associated with solidarity, compassion, open experimentation, and cultures of care.

For this reason, I believe it is imperative to align our structural critiques of the profession within capitalism to those feminist, queer, post-colonial, and indigenous critiques and practices that have been trying to articulate truly different modes of life for, at least, the past five decades. Rather, it requires inventing a whole new culture sensitive to the co-construction of labor, identity, and environment, and thinking what democratic political infrastructures might be able to underpin this culture.

MDA: Certainly, aligning our critiques to those Manuel mentions is essential. But how do we start to think through and beyond critique into action? When we hear about value in terms of the architect specifically how we ask for compensation, how we determine our working conditions, how we change those conditions when we are dissatisfied with them - we often hear arguments that emphasize the 'power' of the individual to ask for 'what she's worth.' The solution, then, is to 'empower' the architect with a cursory knowledge of the market, which includes basic salary information, and encourage her to negotiate with her employer for a higher wage.

If we are invested in creating any kind of systemic change, this approach to the issue of under-compensation is insufficient for multiple reasons. First, the site of change is the individual 
alone, bolstered solely by her own gumption, negotiating for a higher wage. Second, this approach assumes the primacy of the logic of the market and works within it as opposed to against it. The very process of negotiation implies the acknowledgement of and working within boundaries, which in this case are set by the market and by capitalism writ large.

The fundamental difference between ideological individual 'empowerment' and strategic labor organizing lies in the tactics they espouse to change working conditions. The former leads to an individual compromise, while the latter leads to a collective bargain. In collective bargaining, the needs of the worker drive the demands. And, because in the labor movement there is a recognition that workers' needs often fall outside the logic of the market, these needs are also included as demands in the bargaining process. If architects do not negotiate individually, but rather as a collective, they wield the immense power of withholding their labor until management meets their demands.

Organizing labor gives individuals the strength of the collective in order to effectively fight for their value in the workplace and, ultimately, for the value of their work.

РВ: While I am engaged by the question of the role of architects, architecture, and building in the larger machinations of the liberal economy, the ideas provocative as they may be - about restructuring those systems are simply playing a very, very long game. Here, in the short-yardage situation, the value proposition can be seen in much more tactical terms. Thus, I would like to change the focus, and save the critique of an architect's labor union (which I think is a largely futile effort for many reasons) to a latter exchange.

Manuel correctly identifies that there are systemic challenges to the definition and creation of value of the architect's work in the systems of building. But his argument that professional architects exist, solely, as a means to isolate expertise and control a marketplace is hardly borne out by the related statistics. Market control usually manifests in market power, which means influence and recompense, neither of which characterizes our profession.

However, there is a second, equally important reason that society (and markets) create a professional

"Organizing labor gives individuals the strength of the collective in order to effectively fight for their value in the workplace and, ultimately, for the value of their work." 
class: the need for extreme specialization in complex enterprises, and the need to match that competency with responsibility for decision-making. I doubt, for example, if this exchange were to be taken up by 'The Medical Lobby' there would be a similar description of a doctor's services as best served by 'common structures.' Doctors may suffer from the issues of 'liberal business structures' but they deliver services that are otherwise unavailable to those not trained as doctors and we need them. We have a similar issue in architecture, and I think we need architects as well. Defining that responsibility as broadly as possible is a very potent value proposition for architects, but is largely unconverted by architects themselves in the systems of delivery of buildings. Architectural salaries are low, and laborers unappreciated not because of elitist specialization or even lack of negotiating leverage, but because the market-based inputs to the practice are strongly undervalued. Licensure, despite its manifold difficulties, is the single most powerful leverage point architects have in delivering and converting value to the systems of delivery for a simple reason that we undermine at our great peril: you can't build a building without an architect under the current regulations of today. The systems of delivery for buildings, comprising many billions of dollars of spending yearly, are wildly unpredictable - statistics on 'on time' and 'on budget' delivery vary from depressing to absolutely mortifying.

Thus, both of these ideas - public welfare and project execution - are potent examples of huge potential levers of value conversion that leave the typical plaintive cries of architects ('why doesn't anyone love design, and why don't we get paid more for it?') moot. If the definition of 'the public good' were broader and demonstrable, value would be created and architects could get paid more (and could pay their staffs more as well). If project objectives like budget, schedule, sustainability, design quality, etc., can be defined clearly and architects can deliver on those promises, ditto. In a world increasingly driven by data and metrics, this is an opportunity for architects accordingly.

There are of course much larger socio-economic issues to be wrestled with, and I applaud those of you willing to do so in the hopes of setting a course for our profession in the long term. But meanwhile, on the ground, the systems that coalesce to make buildings are shifting under all sorts of influences - neoliberal capital flows, industrialized construction, the advent of data-driven decision-making - and I think we need to deploy shorter term tactics like the ones described above and make sure we're at the table when the larger questions are called down the line. Maybe along the way we can create a more sustainable, valued profession that gets that seat at that table.

DH: Phil's comment has important thoughts to reckon with, but two things strike me. First, the architect is presented as a sort of monolithic 'producer,' which 
might be representative of the client or market's view of the practice, but this elides the problems of how the architect's labor itself is valued, and obscures a bit how the product is constituted of the labor of many workers.

"I do agree that being more specific about what architects do would be a step in the right direction. But this requires acknowledging that the items we might list under our specialists' accountability are not merely market-inputs, but political battlegrounds."

The second thought is, within that workflow, some of the factors Phil mentioned are points at which there is value to be found in new kinds of labor. One such case is 'data-driven' design, and expanding that, workflow automation. The role of the architect in many new computational design frameworks is to determine factors that will have an influence on the behavior of an automated design system, and then to assess and decide (based on a whole other register of 'values') which outcomes are preferable in a given design context. This means that architectural labor could include engagements in a wider range of tasks, where the architect's own values - perhaps more than their design skills or ability to manage processes of production - are a determining factor in the value produced for a client.

My concern for this scheme is that it also doesn't answer the question of the value of architectural labor, so much as broadening the kind of work that might fit. However, it might open the possibility of showing how the value produced for a client can be associated with a process that might be more amenable to such an analysis, though there's more to explore in that regard.

MS: While I agree that we need solutions to address the urgencies of the here-and-now, I also think this need not preclude more long-range and in-depth discussions - indeed, I think one cannot ultimately work without the other.

With regards to the division of specialist labor in a complex modern society, my argument isn't that we have accumulated too much power - if that were the case our monetary rewards would be higher. My argument is that we operate in a system that only rewards those who seek 
to accumulate monetary power by any means necessary (aka capitalist market competition). It is not for our great ethical magnanimity that we have refused to engage in the cannibalistic game of capital and thus accrue this power, but because our profession began as an aristocratic/bourgeois gentleman's endeavor from the very beginning. Architects didn't need the power, because they already had it, at least in a structural sense - they were more connected to capital than labor.

The parallel between the architectural and medical professions is welcomed, but needs two important qualifications. The complex modern enterprises require (indeed, are premised on) extreme specialization and clear accountability; and how this specialization/accountability is channeled is an eminently political - not technical - question. Thus, suggesting that medical services are better served by non-common structures (I assume you're thinking of individual liberal professionals, or private companies, or both), plays directly into the view of specialization as most efficiently honed through market competition. We need to find other ways of accounting for the complex specialization required by modern enterprises; ways that understand that 'health' itself (or any other value) cannot be properly measured through the frameworks of classical or neoliberal political economy.

I do agree that being more specific about what architects do would be a step in the right direction. But this requires acknowledging that the items we might list under our specialists' accountability are not merely market-inputs, but political battlegrounds. Otherwise, we would get a completely de-politicized view of the market. Markets are configured by certain actors and institutions that care not about 'results' (even in an altruistic sense), but rather only their share of the pie.

PD: I want to emphasize how much I think we should pay attention to David's contribution that highlights how, in the present day of information and data hegemony, architecture and architecture knowledge might establish value. Manuel's analysis of capitalism's fundamental shaping of our discipline doesn't, I believe, preclude effectiveness now in some way: it is a reminder that when we seek remedies, we must have the full, long plan in mind and not indulge in misguided things that incidentally reinforce neoliberalism. But I do agree with Phil that we can operate differently in the here and now. At worst, the suggested remedies (only) make immediate problems clear and actionable even though they don't help overthrow the system. At best, the remedies are part of the process of deconstructing that system. What Phil says about how we can work on the most obvious aspect of our market pricing (results) just makes such obvious sense to me and the Lobby has been arguing this 
from the start: our need to make known (and act on) our long-term care for the built environment. But I wonder if the other one of the determinants of market value (scarcity) is addressable - with unions, which Marianela is proposing, being a key. Unions could not, obviously, negotiate the payment contract that owners offer architects, but it could be that unions change the model of architectural work, and do so in a way that is visibly clear to the clients: work that is done by people who have committed to their métier. No film director is embarrassed to say that she only hires union workers; in fact, she'd never boast that she made a film with only non-union help (too 'amateur').

I personally question Phil's belief that licensure is the only or main thing we architects have to secure our value. Certification could ensure expertise and we would immediately be judged by the specifics of our strengths and interests instead of all being dumped wholesale into the 'too expensive' or 'too self-absorbed' bin. Just wondering. But this is not the place for that debate. ARQ

\section{Architecture Lobby}

<info@architecture-lobby.org>

The Architecture Lobby, Inc. is an organization of architectural workers advocating for the value of architecture in the general public and for architectural work within the discipline. It believes that the work architects do - aesthetic, technical, social, organizational, environmental, administrative, fiduciary - needs structural change to be more rewarding and more socially relevant. As long as architecture tolerates abusive practices in the office and the construction site, it cannot insist on its role in and for the public good. 